COLO-HEP-371

hep-th/9607011

June 1996

\title{
A note on brane tension and M-theory
}

\author{
S. P. de Alwis内 \\ Department of Physics, Box 390, University of Colorado, Boulder, CO 80309
}

\begin{abstract}
We point out that some M-theory results for brane tension, can be derived from Polchinski's formula for D-brane tension. We also argue that this formula determines gravitational and gauge couplings in the low energy but quantum exact effective action.
\end{abstract}

\footnotetext{
1e-mail: dealwis@gopika.colorado.edu
} 
Polchinski's formula for the tensions of D-branes [1] states that the tension $T_{p}$ of a D (p)-brane is given by,

$$
T_{p}^{2}=\frac{2 \pi}{2 \kappa^{2}}\left(4 \pi^{2} \alpha^{\prime}\right)^{3-p}
$$

where $1 / 2 \pi \alpha^{\prime}$ is the fundamental string tension and $2 \kappa^{2}$ is (16 times) Newton's constant in 10 dimensions. Now since type IIA string theory has only even $p$ and type IIB only odd $p$ D-branes one might wonder whether the even and odd series of the above formula should be treated separately. However since the two series are related by T-duality one should take this to be one series with one ten dimensional gravitational coupling. This is clarified by recent work of Green, Hull, and Townsend [2] who find from T-duality that

$$
T_{p-1}=2 \pi \alpha^{1 / 2} T_{p}
$$

in agreement with (0.1). It is curious that this relation implies that the (p-1)-brane tension can be obtained from that of the p-brane by compactifying on a circle with the self dual (physical) radius.

Note that $(0.2)$ implies $T_{p}=\left(4 \pi^{2} \alpha^{\prime}\right)^{3-p} T_{6-p}$. Substituting in the brane quantization rule [3]

$$
2 \kappa^{2} T_{p} T_{6-p}=2 \pi n, \quad n \in \mathbf{Z}
$$

we get, for $n=1$, equation (0.1). In other words the T-duality result plus the quantization condition gives Polchinski's formula for D-brane tensions.

Now when $p=1$ we have the D-string of type IIB theory and by $S L(2, Z)$ duality [5] it has tension $g^{-1}\left(2 \pi \alpha^{\prime}\right)^{-1}$, where the second factor is the fundamental string tension, and the relation gives a definition of the effective string coupling which for weak coupling takes the form $g=<e^{\phi}>+\ldots$ where $\phi$ is the dilaton. Hence we have from (0.1) a relation between $\kappa$ and $\alpha^{\prime}$,

\footnotetext{
${ }^{1}$ It should be noted that one needs to replace the non-standard $\alpha^{\prime}$ in [2] according to $\alpha^{\prime} \rightarrow 4 \pi^{2} \alpha^{\prime}$ to get the usual definition.

${ }^{2}$ For a review see 4 .

${ }^{3}$ Actually as shown in [7] [8] one need not assume $S L(2, Z)$ to get this.
} 


$$
2 \kappa^{2}=(2 \pi)^{3}\left(2 \pi \alpha^{\prime}\right)^{4} g^{2}
$$

Let us now discuss the correspondence with M-theory results. Putting $T=1 / 2 \pi \alpha^{\prime}$ the fundamental string tension, we find from (0.1) and (0.4).

$$
\frac{T^{2}}{T_{3}}=\frac{1}{\left(2 \pi \alpha^{\prime}\right)^{2}} \frac{\sqrt{2 \kappa^{2}}}{\sqrt{2 \pi}}=2 \pi g
$$

Similarly one may obtain $T^{3} / T_{5}=(2 \pi)^{2} g$. But these are precisely the formulae obtained by Schwarz [6] using M-theory. Here they have been obtained purely from string theory. This is not so surprising since they relate quantities that are all in type IIB.

Schwarz [6] has also obtained using M-theory arguments, the relation

$$
\frac{T_{5}^{M}}{\left(T_{2}^{M}\right)^{2}}=\frac{1}{2 \pi}
$$

between the M-two-brane and the M-5-brane tension?. We note in passing that a similar relation (0.6) had been obtained earlier by Duff et al [10] by using M-theory quantization conditions at membrane and at 11 dimensional effective supergravity action level, but with the right hand side being twice that given above@. In an appendix we rederive the Duff et al result and find that it actually agrees with (0.6) above (and hence as shown below with the Polchinski quantization condition as well) thus eliminating a potential conflict. We will obtain (0.6) from the purely type IIA relation between the 2-brane and the 4-brane tensions which are determined by (0.1).

The M-theory metric is related to the type IIA one by (see for example [9])

$$
d s_{M}^{2}=g^{4 / 3} d x_{11}^{2}+g^{-2 / 3} d s_{10}^{2}
$$

${ }^{4}$ Note that we have expressed all tensions in the string metric hence the extra factor $g$ as compared with [6] equation (6). Also we have set the axion field to zero for simplicity.

${ }^{5}$ It should be stressed that the ratios on the left hand sides of $(0.5,0.6)$ are dimensionless and hence independent of the metric in which the two tensions are defined.

${ }^{6}$ This is similar to the factor two discrepancy found by Polchinski [1] between his results and an effective action calculation of Harvey and Strominger 11. 
We adopt the convention that the coordinate range of the circular 11th dimension goes over $0-2 \pi \sqrt{\alpha}^{\prime}$ where $1 / 2 \pi \alpha^{\prime}=T$ is now the IIA tension. This then serves to define the effective IIA coupling $g=<e^{\phi}+\ldots>$ in terms of the physical radius of the circle $R_{11}=\sqrt{\alpha^{\prime}} g^{2 / 3}$. In other words $g^{2}=R_{11}^{3} T^{3 / 2}(2 \pi)^{3 / 2}$ is defined (as in the IIB case) in terms of physical quantities. Let us denote the induced string metric on the world sheet in IIA by $\gamma$, and the induced M theory metric on the 2-brane world volume by $\gamma_{M 2}$. The IIA string is obtained from the M-2-brane by double dimensional reduction, i.e. by wrapping one dimesion of the membrane around the circle of M-theory. Then from (0.7) we get, $\sqrt{\gamma}_{M 2}=\sqrt{\gamma}$ and hence $T_{2}^{M} 2 \pi \sqrt{\alpha}^{\prime}=T=1 / 2 \pi \alpha^{\prime}$ giving

$$
T_{2}^{M}=1 /(2 \pi)^{2}\left(\alpha^{\prime}\right)^{\frac{3}{2}}
$$

Now the relations between the two induced metrics on the 2-brane are given (using (0.7)) by $\sqrt{\gamma_{M 2}}=g^{-1} \sqrt{\gamma_{2}}$. The IIA 2-brane is obtained by simple dimensional reduction, and one obtains [9] the relation $T_{2}=g^{-1} T_{2}^{M}$. Using the expression $T_{2}^{2}=(2 \pi)^{3} \alpha^{\prime} / 2 \kappa^{2}$ from (0.1) and equation (0.8) we get again (0.4) except that now $g$ and $\alpha^{\prime}$ are defined as above in the IIA theory.

The 4-brane is obtained from the M-theory 5-brane by double dimensional reduction [9]. Using again (0.7) we have for the world volume densities, $\sqrt{\gamma}_{M}^{5}=g^{-1} \sqrt{\gamma^{5}}$ and using, for consistency, the coordinate radius $\sqrt{\alpha^{\prime}}$ as before, we have $T_{4}^{A}=g^{-1} T_{5}^{M}\left(2 \pi \sqrt{\alpha^{\prime}}\right)$. Using the above relations between IIA tensions and M-tensions, Polchinski's formula (0.1), and (0.4), we get

$$
T_{5}^{M}=(2 \pi)^{-5} \alpha^{\prime-3}
$$

From (0.8) and (0.9) the M-theory relation (0.6) is recovered].

To go further let us use the quantization relation of M-theory,

$$
2 \kappa_{11}^{2} T_{2}^{M} T_{5}^{M}=2 \pi n, \quad n \in \mathbf{Z}
$$

${ }^{7} \mathrm{~A}$ similar derivation of this result has been given by I. Klebanov and A. Tseytlin 12. I wish to thank Igor Klebanov for drawing my attention to these papers. 
Then putting $n=1$ and using the results $(0.8)$ and $(0.9)$, we have,

$$
2 \kappa_{11}^{2}=(2 \pi)^{8} \alpha^{\prime 9 / 2}
$$

This formula (which could also have been obtained by comparing the gravitational actions of M-theory and IIA theory) should be interpreted as fixing the string scale in terms of the fundamental M-theory scale, but for notational convenience we will continue to express everything in terms of $\alpha^{\prime}$.

It should be noted at this point that in comparing the expression (0.11) to the string scale one has to be careful as to what metric the string scale is defined in. We have defined the fundamental string length to be $l_{\text {string }} \equiv \sqrt{\alpha}^{\prime}$ in the string metric. This means that in the M-theory metric this has the value (using the conversion factor from the metric (0.7)) $l_{\text {string }}^{M}=g^{-1 / 3} \sqrt{\alpha^{\prime}}$. Hence we have from (0.11) the expression,

$$
l_{11} \equiv\left[2 \kappa_{11}^{2} /(2 \pi)^{8}\right]^{\frac{1}{9}}=g^{1 / 3} l_{\text {string }}^{M}
$$

for the 11 dimensional Planck length. It should be noted also that our definition of the coupling constant (in the discussion after equation (0.7)) is equivalent to the relation $g=R_{11} / l_{\text {string }}^{M}$. This clarifies the relation between our conventions and that of other authors [12], 13].

Now a highly non-trivial M-theory result obtained by Horava and Witten [14] is the value of the (dimensionless) ratio $\left(2 \kappa_{11}^{2}\right)^{2} / \lambda^{6}=(2 \pi)^{-5}$, where $\lambda$ is the $E_{8} \times E_{8}$ gauge coupling in the theory on $\frac{R_{10} \times S_{1}}{Z_{2}}$. To make a connection, let us calculate in type I or I' theory, making the plausible assumption that since these theories are obtained by a certain projection on type II theories the previous results on the gravitational constant hold true. To identify the gauge coupling let us look at the nine-brane action [15, 16] in the limit of flat space. We have (see for example [16] equation (2.19))

$$
T_{9} \operatorname{tr} \operatorname{det}\left[1+\left(2 \pi \alpha^{\prime}\right) F\right]^{1 / 2} \sim T_{9} \frac{\left(2 \pi \alpha^{\prime}\right)^{2}}{4} \operatorname{tr} F^{2}
$$

\footnotetext{
${ }^{8} \mathrm{I}$ wish to thank Igor Klebanov for raising this issue.
} 
Using the value of $T_{9}$ from the Polchinski formula (0.1) we have for the gauge coupling

$$
\lambda^{2}=(2 \pi)^{5 / 2}\left(2 \pi \alpha^{\prime}\right) \sqrt{2 \kappa^{2}}=(2 \pi)^{7} \alpha^{\prime 3} g
$$

The upshot of these calculations is that we have the following equivalent forms of low-energy effective string (M) theory (with gauge fields) in 10 (11) dimensions. Note that because of the BPS argument for the D-brane tensions it is plausible that these are quantum effective actions with the actual physical couplings at the string scale. i.e. they are low energy, but quantum exact, effective actions, which can be used as the initial condition for the RG evolution (after compactification) down to low energies. Even at strong coupling the form of the low energy effective action is determined by general covariance and gauge invariance, and our claim is that the D-brane formula effectively determines the values of the coefficients. (Note that because of the connection between type I (I') and type IIB (A) the coupling $g$ that we have used should be identified with the type I (or I') couplings $g,\left(g^{\prime}\right)$.

Type I:

$$
S=-\frac{1}{(2 \pi)^{7}} \int d^{10} x \sqrt{G}\left[\frac{g^{-2}}{\left(\alpha^{\prime}\right)^{4}} R+\frac{g^{-1}}{4\left(\alpha^{\prime}\right)^{3}} \operatorname{tr} F^{2}+\ldots\right]
$$

Putting $G_{\mu \nu}=g_{H}^{-1} G_{H \mu \nu}$ and $g_{H}=1 / g$ we have, Heterotic $(S O(32))$ :

$$
S=-\frac{1}{(2 \pi)^{7}} \int d^{10} x \sqrt{G_{H}} g_{H}^{-2}\left[\frac{1}{\left(\alpha^{\prime}\right)^{4}} R+\frac{1}{4\left(\alpha^{\prime}\right)^{3}} \operatorname{tr} F^{2}+\ldots\right]
$$

We also have (using (0.11) in the expression for the action in [14]),

M-theory on $R_{10} \times S_{1} / Z_{2}$ :

$$
S=-\frac{1}{(2 \pi)^{8}\left(\alpha^{\prime}\right)^{9 / 2}} \int d^{11} x \sqrt{G_{M}} R_{M}-\sum_{i} \frac{1}{4} \frac{1}{(2 \pi)^{7}\left(\alpha^{\prime}\right)^{3}} \int_{M_{i}} \operatorname{tr} F_{i}^{2}+\ldots
$$

Transforming to the heterotic metric $G_{M m n}=g_{H}^{4 / 3}\left(d x^{11}\right)^{2}+g_{H}^{-2 / 3} G_{\mu \nu} d x^{\mu} d x^{\nu}$, we get, Heterotic $\left(E_{8} \times E_{8}\right)$ :

$$
S=-\frac{1}{(2 \pi)^{7}} \int d^{10} x \sqrt{G} g_{H}^{-2}\left[\frac{1}{\left(\alpha^{\prime}\right)^{4}} R+\frac{1}{4\left(\alpha^{\prime}\right)^{3}} \sum_{i} \operatorname{tr} F_{i}^{2}+\ldots\right]
$$


Finally putting $G_{\mu \nu}=g_{H} G^{\prime}{ }_{\mu \nu}, g^{\prime}=1 / g_{H}$ we have, Type I':

$$
S=-\frac{1}{(2 \pi)^{7}} \int d^{10} x \sqrt{G^{\prime}}\left[\frac{g^{\prime-2}}{\left(\alpha^{\prime}\right)^{4}} R+\frac{g^{\prime-1}}{4\left(\alpha^{\prime}\right)^{3}} \sum_{i} \operatorname{tr} F_{i}^{2}+\ldots\right]
$$

The point of the above expressions is that we have expressed the low energy couplings in terms of the two physical quantities; the D-string tension or 11 dimensional physical radius, and the fundamental string tension. We also see that the value of the 10 dimensional type I' gauge coupling as determined in (0.14) is consistent with the Horava-Witten calculation [14] and heterotic-type I' duality. Alternatively if we had assumed the latter duality we could have determined the M-theory gauge coupling.

There is also another $\mathrm{M}$ theory number that can be fixed by our arguments. This is the coefficient of the purely gravitational Green-Schwarz term in the M theory action (equation (3.12) of the second paper in [14] or equation (3.14) of [10] which was left undetermined in [14]). In [10] this coefficient is given as the $\mathrm{M}$ theory membrane tension $T_{2}^{M}$. Using our value for this (0.8) we get (note that in the expression below $R$ is the curvature two form and $C_{3}$ is the three form field of $\mathrm{M}$ theory)t

$$
\frac{1}{(2 \pi)^{2} \alpha^{\prime 3 / 2}} \int C_{3} \wedge \frac{1}{(2 \pi)^{4}}\left[-\frac{1}{768}\left(\operatorname{tr} R^{2}\right)^{2}+\frac{1}{192} \operatorname{tr} R^{4}\right] .
$$

On compactifying on a circle of coordinate radius $\sqrt{\alpha^{\prime}}$ in accordance with our convention (note that there are no metric factors in this topological term), we get the correct expression with the right numerical factor for the corresponding expression in the type IIA string (see [10] and references therein). The coefficient above may be expressed in terms of the $11 \mathrm{D}$ gravitational constant using (0.11) and we find $\left[(2 \pi)^{2} / 2 \kappa_{11}^{2}\right]^{1 / 3}$. This gives a numerical coefficient which is a factor of $1 / 3$ times the value given in [14]. At first sight this appears to conflict with the requirement of anomaly cancellation, however it may be the case that there is an alternative way of cancelling the anomalies in the theory of

\footnotetext{
${ }^{9} \mathrm{I}$ wish to thank E. Witten for suggesting this check.

${ }^{10}$ Our conventions are the same as in [10]. In particular our three form field $C_{3}$ is related to that of 114 by $C_{3}=\sqrt{2} C_{3}^{H W}$.
} 
To end this note let us explore the consequences of the speculation that M-theory/nonperturbative string theory effects actually pick the point $g=g_{H}=g^{\prime}=g_{H}^{\prime}=1$ since in some sense this is the most symmetrical choice. The ten dimensional gravitational and gauge couplings are then given by

$$
2 \kappa^{2}=(2 \pi)^{7} \alpha^{\prime 4}, \quad \lambda^{2}=(2 \pi)^{7} \alpha^{\prime 3}
$$

giving the usual relation $2 \kappa^{2}=\alpha^{\prime} \lambda^{2}$ for heterotic strings [17], [18]. It should be noted that the argument in [18] which depends only on the identification of the leading singularity in the operator product expansion of two vertex operators for gauge fields would be independent of world sheet topology and hence should be valid at least to all orders in perturbation theory. The overall normalizations, which in the case of weak coupling perturbation theory are given by $2 \kappa^{2}=\frac{1}{2} g^{2}\left(2 \alpha^{\prime}\right)^{4}, \lambda^{2}=\left(2 \alpha^{\prime}\right)^{3}$ [17], are however changed.

Now Witten [19] has recently argued that strong coupling physics gives a new perspective on the so-called string scale problem (For recent reviews see [20].). Let us see how this works out in our case. 12 The four dimensional couplings are,

$$
G_{N}=\frac{(2 \pi)^{6}}{8} \frac{\alpha^{\prime 4}}{V}, \quad \alpha_{G U T} \equiv \frac{\lambda_{4}^{2}}{4 \pi}=\frac{1}{2}(2 \pi)^{6} \frac{\alpha^{\prime 3}}{V}
$$

Note that the gauge coupling can be small even though the string coupling is unity, if the compactification scale $V^{1 / 6}$ is large compared to the natural scale $2 \pi \sqrt{\alpha}$. The relation between the gravitational and gauge coupling constants is the same as in weak coupling (i.e. $G_{N}=\frac{1}{4} \alpha_{G U T} \alpha^{\prime}$ ). Nevertheless, because of the change in the relation between $\alpha^{\prime}$ and $\alpha_{G U T}$ (coming from the overall factors of $2 \pi$ ), we have a factor of $\simeq 5$ improvement over the weak coupling result. Specifically, eliminating $\alpha^{\prime}$ from (0.22) we have $G_{N}=\alpha_{G U T}^{4 / 3}(2 V)^{1 / 3} / 16 \pi^{2}$. If we assume that the compactification scale is given by the 'observed' unification scale, i.e. $V^{-1 / 6}=M_{G U T}=2-3 \times 10^{16} \mathrm{GeV}$ and put $\alpha_{G U T}^{-1}=25$

\footnotetext{
${ }^{11}$ This matter is currently under investigation.

${ }^{12}$ For further explorations of these phenomenological issues along the lines of [19], see [21], [22].
} 
at that scale [23], we get a Planck mass of $2-3 \times 10^{18} \mathrm{GeV}$, which is still a factor of 4-5 too small. However it is not clear what significance this has, since the identification of the $V^{1 / 6}$ with the unification scale is just an order of magnitude estimate and may be off by factors of $2 \pi$ etc.. In any case there is still the question of why the compactification volume is a factor 10 larger than the natural volume of string compactification $\simeq\left(2 \pi \sqrt{\alpha}^{\prime}\right)^{6}$ (which is what one has for four dimensional strings). Of course the length scales differ only by a factor of $\simeq 1.5$ !

\section{Acknowledgments}

I wish to thank Joe Polchinski and John Schwarz for very useful discussions, and E. Witten for a very useful e-mail message. This work is partially supported by the Department of Energy contract No. DE-FG02-91-ER-40672.

\section{Appendix}

In this appendix the 11D supergravity quantization condition of 10 is rederived and shown to be in agreement with the $(0.6)$ and hence (indirectly) also with $(0.1)[3$. The argument proceeds by using Dirac quantization at the membrane level and then at the $11 \mathrm{D}$ effective action level.

Let us consider the integral of $K_{4}=d C_{3}=d C_{3}^{\prime}$ over a 4-sphere where the two guage fields are used respectively on the upper and lower hemispheres. Then

$$
\begin{aligned}
T_{2} \int_{S_{4}} K_{4} & =T_{2} \int_{S_{4}^{+}} d C_{3}+T_{2} \int_{S_{4}^{+}} d C_{3}^{\prime} \\
& =T_{2} \int_{S_{3}}\left(C_{3}-C_{3}^{\prime}\right)=2 \pi n, \quad n \in \mathcal{Z} .
\end{aligned}
$$

The second equality follows from Stokes' theorem and the last from the requirement that the membrane action should give a well defined quantum theory. Let us now consider the manifold $M_{12}=S^{4} \times S^{4} \times S^{4}$. Using the above result with $n=1$ we get,

$$
\frac{1}{12 \kappa_{11}^{2}} \int_{S^{4} \times S^{4} \times S^{4}} K_{4} \wedge K_{4} \wedge K_{4}=\frac{3 !}{12 \kappa_{11}^{2}}\left(\frac{2 \pi}{T_{2}}\right)^{3}
$$

\footnotetext{
${ }^{13}$ I wish to thank M.J. Duff and R. Minasian for discussions.
} 
On the other hand from Stokes' theorem, and the condition that the topological term in the $11 \mathrm{D}$ supergravity action be quantum mechanically consistent, we get for the left hand side of the above equation,

$$
\begin{aligned}
\frac{1}{12 \kappa_{11}^{2}}\left[\int_{M_{12}^{+}} d C_{3} \wedge K_{4} \wedge K_{4}+\int_{M_{12}^{-}} d C_{3}^{\prime} \wedge K_{4} \wedge K_{4}\right] & =\frac{1}{12 \kappa_{11}^{2}} \int_{M_{11}}\left(C_{3}-C_{3}^{\prime}\right) \wedge K_{4} \wedge K_{4} \\
& =2 \pi n
\end{aligned}
$$

Comparing the two expressions we get

$$
\frac{(2 \pi)^{2}}{2 \kappa_{11}^{2} T_{2}^{3}}=m, \quad m \in \mathcal{Z} .
$$

Combining this with the relation $2 \kappa_{11}^{2} T_{2} T_{5}=2 \pi n$ which follows from the membrane quantization condition and the existence of 5-branes, we get $T_{5} / T_{2}^{2}=n /(2 \pi)$.

\section{References}

[1] J. Polchinski, Phys. Rev. Lett. 754724 (1995), hep-th/9510017.

[2] M.B. Green, C.M. Hull and P.K. Townsend, hep-th/9604119

[3] R.I. Nepomechie, Phys. Rev. D31 1921 (1985); C. Teitelboim, Phys. Lett. B167 63.69 (1986).

[4] M. J. Duff, R. R. Khuri and J. X. Lu, Phys. Rep. 259213 (1995).

[5] J. H. Schwarz, Phys. Lett. B360 13 (1995), erratum, ibid. B364, 252

[6] J. H. Schwarz, hep-th/9509148; hep-th/9510086.

[7] C. Schmidhuber, hep-th/9601003.

[8] S. P. de Alwis and K. Sato, hep-th/9601167, Phys. Rev. D53 7187 (1996).

[9] P. Townsend, hep-th/95012062. 
[10] M.J. Duff, J.T. Liu, and R. Minasihan, Nucl. Phys. B452 261 (1995) heoth/9506126.

[11] J. Harvey and A. Strominger, Nucl. Phys. B449 535 (1995).

[12] I. Klebanov and A. Tseytlin, hep-th/9604089 and 9604166.

[13] D. Kabat and P. Pouliot, hep-th/9603127.

[14] P. Horava, and E. Witten, hep-th/9510209, hep-th/9603142.

[15] R. G. Leigh, Mod. Phys. Lett. A4, 2767 (1989).

[16] J. Polchinski, S. Chaudhuri, and C. Johnson, hep-th/9602052.

[17] D. Gross, J. Harvey, E. Martinec, and R. Rohm, Nucl. Phys. B267 75 (1985).

[18] P. Ginsparg, Phys. Lett. B197 139 (1987).

[19] E. Witten, hep-th/9602070.

[20] H. P. Nilles, hep-th/9601241; K.R. Dienes, hep-th/9602045; F. Quevedo, hepth/9603074.

[21] T. Banks and M. Dine, hep-th/9605136.

[22] E. Caceres, V.S. Kaplunovsky, and I. M. Mandelberg, hep-th/9606036.

[23] See for example P. Langacker and M. Luo, Phys. Rev. D44 817 (1991). 